\title{
Awareness and forgetting of facts and agents
}

\author{
Hans van Ditmarsch \\ Dept. Logic \\ University of Sevilla \\ Sevilla, Spain \\ Email:hans@cs.otago.ac.nz
}

\author{
Tim French \\ Computer Science and Software Engineering \\ University of Western Australia \\ Perth, Australia \\ Email:tim@csse.uwa.edu.au
}

\begin{abstract}
We propose various logical semantics for change of awareness. The setting is that of multiple agents that may become aware of facts or other agents, or forget about them. We model these dynamics by quantifying over propositional variables and agent variables, in a multi-agent epistemic language with awareness operators, employing a notion of bisimulation with a clause for 'same awareness'. The quantification is over all different ways in which an agent can become aware (or forget). Logics for change of awareness combine well with logics for informational change, as when a public announcement simultaneously makes you aware of an issue ('a plane just crashed on Schiphol Airport').
\end{abstract} tems

Keywords-awareness; knowledge; dynamics; multi-agent sys-

\section{INTRODUCTION}

Becoming aware of facts: When modelling uncertainty in a multi-agent system you only pay attention to the relevant facts. You may then become aware of more facts, and integrate those in the system: a refinement operation. It is not obvious how to do this in epistemic logic! For example, when an agent $i$ is uncertain of the value of some fact (atom / propositional variable) $p$, a two-state structure suffices to represent that uncertainty where $p$ is true in one state and $p$ is false in another state, namely as follows:

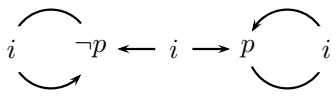

It may now occur that subsequent information about the agent's uncertainty comes to light. Apart from fact $p$, another fact $q$ is also relevant - the modeller and curiously enough also the agent become aware of $q$. One way in which the agent may become aware of $q$ is as follows:

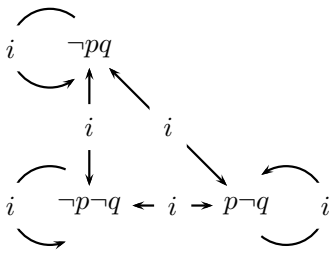

We would like to have a logical operation describing this transition. But here we have a problem. A logic for such transitions would have background paramaters 'set of propositional variables' (facts) and 'set of agent variables', both in the language and in the structures on which the language is interpreted. The first figure is then slightly informal in that no value for $q$ is given in the states. For example, $q$ may be true in both states. That choice would make the transition to the resulting figure very unsatisfactory: the agent would initially know that $q$-we should apparently ignore that but how?-and after becoming aware of $q$ it no longer knows $q$. Any other choice for the initial values of $q$ would create a similar conflict! The solution is to regard the initial values of $q$ as 'don't care': something the agent is unaware of. The transition can then be visualized as in Figure 1-what the agent is unaware of is between parentheses, the other transition is explained later. We propose a logic $(i)$ in which

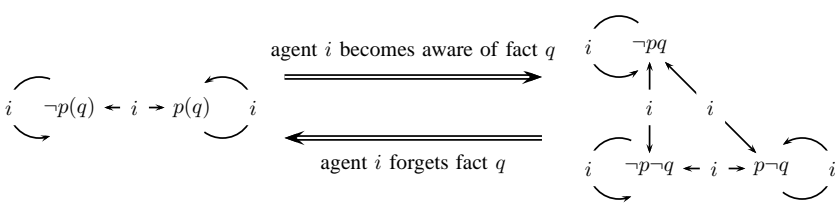

Figure 1. Agent $i$ becomes aware of or forgets fact $q$

the value of $q$ is initially irrelevant, i.e., something the agent is unaware of; ( $i i)$ in which the depicted transition makes the agent aware of $q$, wherein any increased structure expressing uncertainty about $q$ is allowed; and ( iii) in which the agent is then aware of $q$.

Comparison to standard research on awareness: Static awareness in our approach is according to the semantically flavoured proposals by [2], [3], [4]-the extraordinarily rich and exceptionally well-written [2] certainly inspired us along our chosen path. (There is no relation between our approach and syntactically flavoured proposals for awareness, that model 'limited rationality' of agents, such as another approach also pursued in [2] and recent work like [5], [6].) Dynamic awareness is modelled by a bisimulation quantification on structures incorporating awareness, over the variable expressing the newly relevant fact. For bisimulation quantification see [7], [8], this concerns a further generalization of propositional quantification à la Fine [9]. We think that our ideas on dynamic awareness are novel. We are newcomers in the area of awareness logics, and have studied some of the (even) more recent literature on the topic 
[10], [11], [12]. Only Hill's [11] addresses the dynamics of awareness, but rather different from ours: there, the result of becoming aware of a new fact should be ignorance about that fact. On the other hand...: comparison of different levels of awareness is quite standard in the literature-merely not having dynamic operations to go from one to another level. It is remarkable that the relation between the different static ways of unawareness in Heifetz et al.'s [4] can just as well be described in terms of bisimulation quantification. For example, take $S_{\{p\}}$ and $S_{\{p, q\}}$ in their Figure 2 [4, p.86]: $S_{\{p, q\}}$ sums up all different ways in which an agent only aware of $p$, as in $S_{\{p\}}$, can become aware of $q$ (and our running example, above, can indeed be found among those). The notion of bisimulation seems a more succinct, technical tool to express the same. But it inspired our research to see this correspondence with the acclaimed [4].

Forgetting facts: Apart from becoming aware of a fact, the agent may become unaware of a fact. This can be for voluntary and involuntary reasons: for the purpose of abstraction, to focus computational resources on 'more relevant' facts, by gradually things slipping from the mind, or because information is received that all beliefs about that fact are unreliable. Becoming unaware can also be modelled by a bisimulation quantification, and we have pictured this as well in Figure 1.

Becoming aware of other agents: And apart from becoming aware of a fact, an agent can also become aware of another agent in the system, and of the uncertainties of that agent about facts (and about other agents, including the observing agent). In other words, not all agents may be visible to a given agent at a given moment. We provide a similar operation for 'the agent becomes aware of agent $i$, including its dual where visible agents slip into oblivion: 'the agent becomes unware of (forgets) agent $i$ '. This employs bisimulation quantification over an agent variable-an idea originally proposed in [13] as far as we know, but so far not employed in dynamic epistemics.

Figure 2 gives an example. The initial state of information is a slight adjustment of the previous example, as there is now another agent $j$ as well, of which agent $i$ is unaware and who is knowledgable about $p$. (But who is not introspective, as he is not aware of himself.) The figure depicts how agent $i$ can become aware of agent $j$, and also the dual option of forgetting. The unaware atoms and agents are in parentheses. An arrow with two agent variables stands for two arrows.

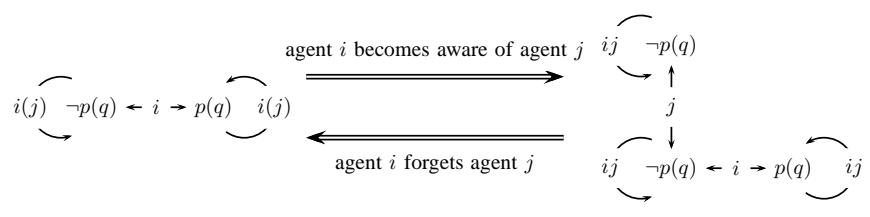

Figure 2. Agent $i$ becomes aware of and forget about other agent $j$. On the right, if agent $j$ knows that $p$ is false, he is uncertain if agent $i$ knows that.
So far, the examples addressed change of awareness that is the same for all states and for all agents. We call this public global awareness. But awareness change can also be different for each agent (individual global awareness), and even in each state (individual local awareness, the common assumption in works like [2]). We present natural generalizations of our ideas to those settings.

Implicit and explicit knowledge: We did not distinguish the modeller becoming aware of new facts or agents from the agents gaining that awareness. This is because we see 'becoming aware' as a structural refinement, where our starting point is an initial minimal structure. 'Unaware' means 'don't care', and we are not interested in the structure of unawareness. This seems to be different from a typical economist's perspective, where the focus is more on unawarenes than on awareness [3], [12]. It also has as unintended consequence that the usual distinction between implicit and explicit knowledge is meaningless in our setting. Explicit knowledge is implicit knowledge of concepts of which you are aware. In the initial two-state structure in Figure 1 agent $i$ implicitly knows $q$. After becoming aware of $q$, he has become explicitly ignorant about $q$ ! In other words, becoming aware does not mean that implicit knowledge will become explicit, an intuitively appealing requirement for awareness change. (Despite this, we have chosen to let the standard distinction persist in our approach as a simplifying technical device.)

In case this is seen as a restriction: we can overcome the restriction in two steps. First, instead of bisimulation quantification, the dynamics that we present could with some reason also be based on Fine's propositional quantification [9] (this might even have advantages for the axiomatization). This only changes the denotation of the propositional variable, without changing anything else of the model structure. This does not solve the problem yet, clearly. Second, suppose we now as well disallow propositional quantification but only allow moving variables around from unaware to aware and vice versa: this keeps all factual and epistemic structure of our models constant, and, presto, all implicit knowledge can now become explicit. That this can be accomplished so easily we see as a real advantage of our framework: it provides tools to model awareness change and can be tailored according to modellers' needs and system requirements. Figure 3 pictures agent $i$ becoming aware of $q$ such that her implicit knowledge that $p$ and $q$ are not both true becomes explicit:

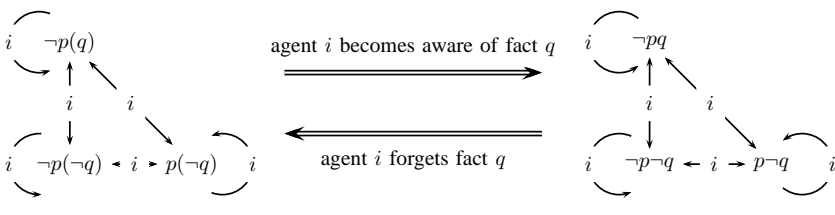

Figure 3. Making implicit knowledge explicit 
Awareness and information: The logics for change of awareness combine well with logics for change of knowledge [14]. A typical example is the announcement of a fact $p$ of which the agents were unaware. The announcer addresses an issue, the truth about $p$, simultaneously with revealing the truth about that issue. A surprising result is that arbitrarily complex informational change (private announcements, suspicions, ...: in fact anything representable by an action model [15]) can be seen as the public announcement of a true fact of which the agents were unaware. For lack of space, this matter will not be addressed in here, see [1] for the (isolated) result.

Quantifying over awareness change: We should emphasize that our logics model quantification over all possible ways in which an agent can become aware of a concept (i.e., fact or other agent), but do not model specific awareness change. In other words, it is not important how the agent gained this awareness. Or in yet other words, it is a logic about speculation over all future scenarios if we were to become aware of some relevant facts or other players later. But surely, any conclusions that can be drawn from that already are of great interest not just to academic pursuits of economics but also, currently, to the real economy.

In Section II we introduce epistemic awareness models wherein knowledge and awareness are encoded. An appropriately expanded notion of bisimulation is also introduced there (apart from atoms, back, and forth, there is a fourth clause involving awareness). In Section III we present the Logic of Public Global Awareness (LPGA) and the language $\mathcal{L}_{0}$ : the awareness of facts and other agents at a given moment is the same for all agents. In Section IV we present the Logic of Individual Global Awareness (LIGA) and the language $\mathcal{L}$ : the level of awareness can vary between agents, but is the same in all states. In Section V we summarily present the Logic of Individual Local Awareness (LILA) (also based on $\mathcal{L}$ ), wherein for each agent and each state the level of awareness may vary, and some issues for further research.

\section{STRUCTURES}

Given are a countably infinite set of propositional variables (facts) $P$ and a countably infinite set of agents $N$. As we are also modelling 'becoming aware of an agent', any finite number of agents would be insufficient: whatever the finite number of agents in one's company, someone else can always turn up at any stage! The sets $P$ and $N$ are disjoint. The union $P \cup N$ is called the set of concepts. Propositional variables are named $p, q, r$, possibly indexed or quoted, and agent variables are named $i, j, k$, possibly indexed or quoted. For any set $X$, write $X+x$ for $X \cup\{x\}$ and write $X-x$ for $X \backslash\{x\}$. Write $\bar{Y}$ for $X \backslash Y$ and similarly $\bar{x}$ for $X-x$.
Epistemic awareness model: An epistemic awareness model $M=(S, R, \mathcal{A}, V)$ for $N$ and $P$ consists of a domain $S$ of (factual) states (or 'worlds'), an accessibility function $R: N \rightarrow \mathcal{P}(S \times S)$, an awareness function $\mathcal{A}: N \rightarrow S \rightarrow$ $\mathcal{P}(P \cup N)$ and a valuation function $V: P \rightarrow \mathcal{P}(S)$. For $R(i)$ we write $R_{i}$ and for $\mathcal{A}(i)$ we write $\mathcal{A}_{i}$; accessibility function $R$ can be seen as a set of accessibility relations $R_{i}$, and $V$ as a set of valuations $V(p)$. A pointed epistemic awareness model $(M, s)$ is an epistemic awareness state.

Given an agent $i$ and a state $s$, a fact in $\mathcal{A}_{i}(s)$ (i.e., an element of $\mathcal{A}_{i}(s) \cap P$ ) is called relevant (for that agent, given that state), and a fact in $P \backslash \mathcal{A}_{i}(s)$ is called irrelevant. Similarly, an agent in $\mathcal{A}_{i}(s)$ is called visible, and an agent in $N \backslash \mathcal{A}_{i}(s)$ is called invisible.

The awareness function $\mathcal{A}$ satisfies public global awareness iff the value of $\mathcal{A}$ is the same for all agents and for all states. Slightly abusing the (mathematical) language, we then write $\mathcal{A}(S)$ for the set of (globally) relevant facts and visible agents. The awareness function $\mathcal{A}$ satisfies individual global awareness iff the awareness is the same in all states, but maybe different between agents. We then write $\mathcal{A}_{i}(S)$ for the set of (globally) relevant facts and visible agents for that agent. If the awareness may be different for all agents and in all states (the usual assumption in the literature) we call it individual local awareness.

Epistemic uncertainty over awareness is commonly ruled out (there are exceptions, such as [12]): an agent is supposed to know whether she is aware of a fact or of another agent. This condition corresponds to the property on epistemic awareness models of no uncertain awareness defined as (universal quantification over all variables in) "If $(s, t),(s, u) \in R_{i}$, then $\mathcal{A}_{i}(t)=\mathcal{A}_{i}(u)$." If $R_{i}$ is an equivalence relation (to interpret knowledge of an agent), then, if 'no uncertain awareness' is satisfied, the partition induced by $R_{i}$ on the domain is a refinement of the partition induced by $\mathcal{A}_{i}$.

Given an epistemic awareness model $(M, s)$ with awareness function $\mathcal{A}$, we write $(M, s)^{\mathcal{A}_{i}+p}$ for the model that is like $(M, s)$ except for its awareness function $\mathcal{A}^{\prime}$ for which we have $\mathcal{A}_{i}^{\prime}(s)=\mathcal{A}_{i}(s)+p$. When 'no uncertain awareness' holds we assume that $p$ is added for all states in $i$ 's equivalence class, when 'individual global awareness' holds we assume that $p$ is added for all states in the model, and for 'public global' conditions we write $(M, s)^{\mathcal{A}+p}$. Similarly for $(M, s)^{\mathcal{A}_{i}+p}$, and for $(M, s)^{\mathcal{A}_{i}-p}$ (to be used in a definition for forgetting), etc.

Bisimulation: Let two models $M=(S, R, \mathcal{A}, V)$ and $M^{\prime}=\left(S^{\prime}, R^{\prime}, \mathcal{A}^{\prime}, V^{\prime}\right)$ be given. A non-empty relation $\mathfrak{R} \subseteq$ $S \times S^{\prime}$ is a bisimulation, iff for all $s \in S$ and $s^{\prime} \in S^{\prime}$ with $\left(s, s^{\prime}\right) \in \mathfrak{R}$ :

atoms $s \in V(p)$ iff $s^{\prime} \in V^{\prime}(p)$ for all $p \in P$;

aware for all $i \in N, \mathcal{A}_{i}(s)=\mathcal{A}_{i}^{\prime}\left(s^{\prime}\right)$;

forth for all $i \in N$ and $t \in S$, if $R_{i}(s, t)$ then there is a $t^{\prime} \in S^{\prime}$ such that $R_{i}\left(s^{\prime}, t^{\prime}\right)$ and $\left(t, t^{\prime}\right) \in \mathfrak{R}$; 
back for all $i \in N$ and $t^{\prime} \in S^{\prime}$, if $R_{i}\left(s^{\prime}, t^{\prime}\right)$ then there is a $t \in S$ such that $R_{i}(s, t)$ and $\left(t, t^{\prime}\right) \in \mathfrak{R}$.

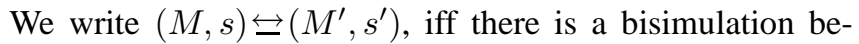
tween $M$ and $M^{\prime}$ linking $s$ and $s^{\prime}$, and we call $(M, s)$ and $\left(M^{\prime}, s^{\prime}\right)$ bisimilar. The novelty of our definition is the clause aware, that requires that bisimilar states have the same level of awareness.

A bisimulation except for fact $p$ satisfies atoms for $P-p$, and aware to the extent that $\mathcal{A}_{i}(s)-p=\mathcal{A}_{i}\left(s^{\prime}\right)-p$. The value of $p$ may vary, including uncertainty of the agents about $p$ and about each other's uncertainty, and the agents may have different awareness of fact $p$. We write

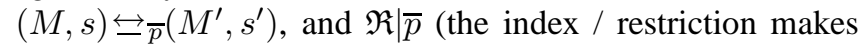
the subset explicit for which the bisimulation should hold). A bisimulation except for agent $i$ satisfies back and forth for $N-i$, and aware to the extent that $\mathcal{A}_{i}(s)-i=\mathcal{A}_{i}\left(s^{\prime}\right)-i$. The accessibility relation and awareness for agent $i$ may vary, including uncertainty of other agents about $i$ 's un-

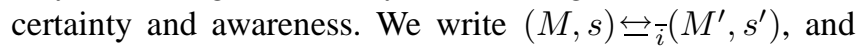
$\mathfrak{R} \mid \bar{i}$. The notion of restricted bisimilarity and its notation generalize in the obvious way to more facts and agents, also in combination.

Awareness bisimulation: The notion of bisimulation will be exactly what we need to capture awareness change under conditions of public global and individual global awareness. It is also effective under conditions of individual local awareness, but in that case too restrictive-now we need a coarser notion of structural similarity to cover all different ways in which an agent can become aware of facts or agents. (After the definition and introduction now to follow, this more complex notion will only be used in Section V.)

Consider the following example: in the actual state $s$ agent $i$ is aware of agent $j$ and of fact $p$, and state $t$ is $i$-accessible from the actual state. In state $t$, agent $j$ is aware of $p$ and $q$. That agent $j$ is also aware of $q$ should leave agent $i$ indifferent, as she was not aware of $q$ in the actual state. Therefore, in case agent $i$ were to become aware of $q$ in state $s$, she should consider it possible that $j$ is unaware of $q$ in that $i$-accessible state $t$. Under conditions of public or individual global awareness this is not a variation we care to consider: if $j$ is aware of $q$ in $t$, then he is already aware of $q$ in the actual state $s$. Clearly, we do not want to change the value of atoms of which agents are aware in the actual state.

This sort of similarity is captured in the following notion, named awareness bisimulation. If two models are awareness bisimilar, they cannot be distinguished (as we will see later) by the fragment of the language of which the agents are aware: therefore, they are described by the same explicit knowledge. The notion is somewhat involved, and strictly based on the pointed versions of our structures. In the following, we use the notational abbreviation $\mathcal{A}(s)$ for $\lambda i \cdot \mathcal{A}_{i}(s)$, and the abbrevation $N(s)$ for the set of all agents of which some agent is aware in state $s$, i.e., $\left\{j \in N \mid\right.$ there is an $k \in N$ such that $\left.j \in \mathcal{A}_{k}(s)\right\}$.

Let epistemic awareness states $(M, u)=$ $((S, R, \mathcal{A}, V), u)$ and $\left(M^{\prime}, u^{\prime}\right)=\left(\left(S^{\prime}, R^{\prime}, \mathcal{A}^{\prime}, V^{\prime}\right), u^{\prime}\right)$ be given. A non-empty relation $\mathfrak{R}^{A} \subseteq S \times S^{\prime}$ (where $A$ in $\mathfrak{R}^{A}$ stands for 'aware') is an awareness bisimulation

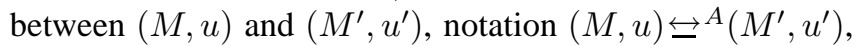
iff $\left(u, u^{\prime}\right) \in \mathfrak{R}^{A}$ and $\mathfrak{R}^{A}=\bigcap_{j \in N(u)} \mathfrak{R}_{j}^{A}[\mathcal{A}(u)]$. We continue by defining $\mathfrak{R}_{j}^{A}\left[\mathcal{A}^{\prime \prime}\right]$ for any $\mathcal{A}^{\prime \prime}: N \rightarrow \mathcal{P}(P \cup N)$ (write $\mathcal{A}_{i}^{\prime \prime}$ for $\mathcal{A}^{\prime \prime}(i)$ ). Let such a $\mathcal{A}^{\prime \prime}$ be given, $s \in S$, and $s^{\prime} \in S^{\prime}$, then $\left(s, s^{\prime}\right) \in \mathfrak{R}_{j}^{A}\left[\mathcal{A}^{\prime \prime}\right]$ iff:

atoms $s \in V(p)$ iff $s^{\prime} \in V^{\prime}(p)$ for all $p \in \mathcal{A}_{j}^{\prime \prime}$; aware for all $i \in \mathcal{A}_{j}^{\prime \prime}, \mathcal{A}_{i}(s) \cap \mathcal{A}_{j}^{\prime \prime}=\mathcal{A}_{i}^{\prime}\left(s^{\prime}\right) \cap \mathcal{A}_{j}^{\prime \prime}$;

forth for all $i \in \mathcal{A}_{j}^{\prime \prime}$ and $t \in S$, if $R_{i}(s, t)$ then there is a $t^{\prime} \in S^{\prime}$ such that $R_{i}\left(s^{\prime}, t^{\prime}\right)$ and $\left(t, t^{\prime}\right) \in \mathfrak{R}_{j}^{A}\left[\mathcal{A}^{\prime \prime} \cap\right.$ $\left.\mathcal{A}^{\prime}(t)\right]$

back for all $i \in \mathcal{A}_{j}^{\prime \prime}$ and $t^{\prime} \in S^{\prime}$, if $R_{i}\left(s^{\prime}, t^{\prime}\right)$ then there is a $t \in S$ such that $R_{i}(s, t)$ and $\left(t, t^{\prime}\right) \in \mathfrak{R}_{j}^{A}\left[\mathcal{A}^{\prime \prime} \cap\right.$ $\left.\mathcal{A}^{\prime}(t)\right]$.

In the back and forth clauses, the relation $\mathfrak{R}_{j}^{A}\left[\mathcal{A}^{\prime \prime} \cap \mathcal{A}^{\prime}(t)\right]$ is inductively assumed to be already defined. Note that $\mathfrak{R}_{j}^{A}\left[\mathcal{A}^{\prime \prime} \cap \mathcal{A}^{\prime}(t)\right]$ is a function from the set of agents to a possibly smaller subset of facts and agents than in $\mathfrak{R}_{j}^{A}\left[\mathcal{A}^{\prime \prime}\right]$, and that these functions are downwardly closed: if $\mathcal{A}_{j}^{\prime \prime}=\emptyset$, then $\mathfrak{R}_{j}^{A}\left[\mathcal{A}^{\prime \prime}\right]=\emptyset$. In finite multi-S5 structures a stable point will be finitely reached in any chain where $\mathfrak{R}_{j}^{A}\left[\mathcal{A}^{\prime \prime} \cap \mathcal{A}^{\prime}(t)\right]=\mathfrak{R}_{j}^{A}\left[\mathcal{A}^{\prime \prime}\right]$. The relation $\mathfrak{R}^{A}$ is indeed an equivalence (proof omitted).

The relation between bisimulation and awareness bisimulation is now as follows. $(i)$ For the model class satisfying public global awareness, awareness bisimulation reverts to bisimulation by way of $\mathfrak{R} \mid \mathcal{A}(S)=\mathfrak{R}^{A}$. (ii) For the model class satisfying individual global awareness we have instead that $\mathfrak{R} \mid \mathcal{A}_{i}(S)=\mathfrak{R}_{i}^{A}\left[\lambda i \cdot \mathcal{A}_{i}(S)\right]$, from which directly follows that $\mathfrak{R}^{A}$ is the intersection of all $\mathfrak{R} \mid \mathcal{A}_{i}(S)$ such that some agent is aware of $i$ somewhere-similarly to above, let $N(S)$ stand for the set of agents of which some agent is aware in some state: $\bigcap_{i \in N(S)} \mathfrak{R} \mid \mathcal{A}_{i}(S)=\mathfrak{R}^{A}$. (iii) Otherwise, as already mentioned, $\mathfrak{R}$ is a refinement of $\mathfrak{R}^{A}$. (Proofs omitted.)

\section{Public GLOBAL AWAREnESS}

Language: Given are a countably infinite set of propositional variables (facts) $P$, and a countably infinite set of agents $N$. The language $\mathcal{L}_{0}$ of public global awareness is defined as

$$
\varphi::=p|\varphi \wedge \varphi| \neg \varphi\left|K_{i} \varphi\right| \exists p \varphi|\exists i \varphi| A \varphi
$$


where $p \in P$ and $i \in N$. By notational abbreviation are defined:

$$
\begin{aligned}
\top & =\exists p(p \vee \neg p) \\
\dot{K}_{i} \varphi & =A \varphi \wedge K_{i} \varphi \\
\dot{\exists} p \varphi & =\neg A p \wedge \exists p(\varphi \wedge A p) \\
\dot{\exists} i \varphi & =\neg A K_{i} \top \wedge \exists i\left(\varphi \wedge A K_{i} \top\right) \\
\dot{\exists} p \varphi & =A p \wedge \exists p(\varphi \wedge \neg A p) \\
\dot{\exists} i \varphi & =A K_{i} \top \wedge \exists i\left(\varphi \wedge \neg A K_{i} \top\right)
\end{aligned}
$$

Construct $K_{i} \varphi$ stands for 'agent $i$ implicitly knows $\varphi$ '. We already pointed out in the introduction that this is a rather tentative phrasing in our setting. Construct $A \varphi$ stands for 'the agents are aware of $\varphi$ ', or rather more strictly, looking ahead to our semantics: 'the visible agents are collectively aware of $\varphi$ '. The curiously non-standard definition of $T$ is to make explicit knowledge of truth possible even if all facts are irrelevant. The meaning of the bisimulation quantifications $\exists p \varphi$ and $\exists i \varphi$ is less intuitive than that of their counterparts that were introduced by abbreviation:

$\begin{array}{ll}\dot{K}_{i} \varphi & \text { agent } i \text { (explicitly) knows } \varphi \\ \dot{\exists} p \varphi & \text { after the agents become aware of fact } p, \varphi \\ \dot{\exists} i \varphi & \text { after the agents become aware of agent } i, \varphi \\ \dot{\exists} p \varphi & \text { after the agents forget fact } p, \varphi \\ \dot{\exists} i \varphi & \text { after the agents forget agent } i, \varphi\end{array}$

Let us explain one of these abbrevations. Explicit awareness $\dot{\exists} p \varphi$ is defined as $\neg A p \wedge \exists p(\varphi \wedge A p)$, which says that the agents are currently not aware of fact $p$, and there is a way to vary the valuation and the awareness of the $p$, such that afterwards $\varphi$ is true and the agents are aware of fact $p$. We have to distinguish universal from existential readings of becoming aware: $\exists p$ says that 'there is a way to become aware of $p$ after which ...', but we need $\forall p$ for 'after any way to become aware of $p$...'.

The semantics of the awareness operator $A$ will be purely syntax-based, namely using the free variables of a formula. These are defined as follows (note that $\operatorname{var}(\varphi) \subseteq P \cup N$ ): $\operatorname{var}(p)=\{p\}, \operatorname{var}(\varphi \wedge \psi)=\operatorname{var}(\varphi) \cup \operatorname{var}(\psi), \operatorname{var}(\neg \varphi)=$ $\operatorname{var}(\varphi), \operatorname{var}\left(K_{i} \varphi\right)=\operatorname{var}(\varphi)+i, \operatorname{var}(\exists p \varphi)=\operatorname{var}(\varphi)-p$, $\operatorname{var}(\exists i \varphi)=\operatorname{var}(\varphi)-i$, and $\operatorname{var}(A \varphi)=\operatorname{var}(\varphi)$.

Semantics: Let $M=(S, R, \mathcal{A}, V)$ be given. We remind the reader that the function $\mathcal{A}$ in the case of public global awareness is constant for all agents and for all states, and that we write $\mathcal{A}(S)$ for the set of relevant facts and visible agents. Below, let the models $M^{\prime}$ have the structure $\left(S^{\prime}, R^{\prime}, \mathcal{A}^{\prime}, V^{\prime}\right)$.
$(M, s) \models p$
$(M, s) \models \varphi \wedge \psi$
$(M, s) \models \neg \varphi$
iff $s \in V(p)$
$(M, s) \models K_{i} \varphi$
$(M, s) \models \exists p \varphi$
iff $(M, s) \models \varphi$ and $(M, s) \models \psi$
iff $(M, s) \not \models \varphi$
iff for all $t:(s, t) \in R_{i} \Rightarrow(M, t) \models \varphi$
iff there is a $\left(M^{\prime}, s^{\prime}\right)$ such that

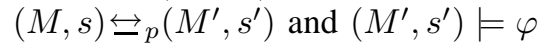
$(M, s) \models \exists i \varphi$
iff
there is a $\left(M^{\prime}, s^{\prime}\right)$ such that
$(M, s) \models A \varphi$

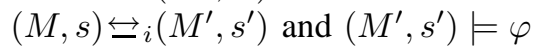
iff $\operatorname{var}(\varphi) \subseteq \mathcal{A}(S)$

The set of validities (and the logic) is called LPGA (Logic of Public Global Awareness). The semantics of all these operators, including $K_{i} \varphi, \exists p \varphi$, and $A \varphi$, is nearly perfectly standard-except for the additional bisimulation requirement with respect to the awareness function. They are the usual suspects - and bring their usual advantages and disadvantages. We could have made different choices. In particular we could have hardwired 'becoming aware' into the semantics just as for explicit knowledge.

We can now explain the curious awareness clauses in becoming aware of and forgetting about other agents. Consider $\dot{\exists} i \varphi$ (the agents forget about agent $i$ ) which stands for $A K_{i} \top \wedge \exists i\left(\varphi \wedge \neg A K_{i} \top\right)$. The requirement $A K_{i} \top$ states that the agent must currently be aware of that agent $i$ for the forgetting to be able to take place. Well, that seems to make sense, you cannot forget something that you are not aware of in the first place. We can pin down that the agents are aware of $p$ by any formula containing the $i$ somewhere (except when bound by a quantifier). But we cannot take, e.g., the formula $A K_{i} p$ for just any fact $p$, as the agents may not be aware of that atom. The requirement $A K_{i} p$ would then be false, even if the agents are aware of $i$ ! By choosing $A K_{i} \top$ this is avoided: using the notational abbreviation for $\top$ this stands for $A K_{i} \exists p(p \vee \neg p)$, and the set of free variables of this formula is just $\{i\}$; the formula $K_{i} \exists p(p \vee \neg p)$ expresses that agent $i$ knows that, if the agents (including $i$ therefore) were to become aware of $p$, tautologies involving it would by all means be true.

Example: Figure 1 models that the agents (namely agent $i$ ) become aware of $q$. Initially, the agent is only aware of $p$. We can now check in the semantics that all of the following hold throughout the initial model: $A p, \neg A q, \dot{\exists} q \dot{K}_{i} \neg(p \vee q)$. The two models in the figure are bisimilar except for fact $q$. In Figure 2 we have that in the initial model, in the (left) state where $p$ is false and relevant and $q$ is true and irrelevant, $\exists j K_{j} \neg p \rightarrow \neg K_{j} K_{i} \neg p$ is true: after the agents become aware of $j$, then if that agent knows that $p$ is false he is uncertain whether agent $i$ knows that. Figure 3 illustrates how implicit knowledge can only become explicit, according to the simpler proposal to follow.

Axiomatization and theory: We have not axiomatized the logic yet, although we have a list of tentative axioms. Among the more obvious principles are $\forall p \quad p \leftrightarrow \perp$ and $\forall p q \leftrightarrow q$, for $q \neq p$. Here we encounter the disadvantages of taking along the usual suspects: bisimulation quantified logics are known to be hard to axiomatize. But they have other desirable theoretical properties that are easier to get. We think it is feasible to show that the logic $L P G A$ is decidable, via a translation into the $\mu$-calculus, employing the techniques of [13]. We also consider the axiomatization with respect to the multi $S 5$ models, where $K_{i} \varphi$ indeed stands for knowledge of $\varphi$, as usual.

Variations: implicit and explicit knowledge: We recall that in this semantics an agent may know $\varphi$ implicitly, but 
may not get to know $\varphi$ explicitly. Consider the semantics again for becoming aware, now (mere semantic sugar) expressed directly:

$(M, s) \models \dot{\exists} p \varphi$ iff there is a $\left(M^{\prime}, s^{\prime}\right)$ such that

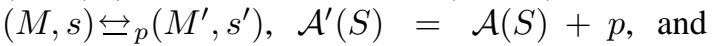
$\left(M^{\prime}, s^{\prime}\right) \models \varphi$.

Consider propositional quantification à la Fine, tentatively expressed with $\equiv_{p}$-as this does not allow variation in awareness we must express that directly in the resulting model:

$(M, s) \models \dot{\exists} p \varphi$ iff there is a $\left(M^{\prime}, s^{\prime}\right)$ such that $(M, s) \equiv_{p}\left(M^{\prime}, s^{\prime}\right)$ and $\left(M^{\prime}, s^{\prime}\right)^{\mathcal{A}+p} \models \varphi$.

Now remove even the Fine quantification and we get

$(M, s) \models \dot{\exists} p \varphi$ iff there is a $\left(M^{\prime}, s^{\prime}\right)$ such that $(M, s)=\left(M^{\prime}, s^{\prime}\right)$ and $\left(M^{\prime}, s^{\prime}\right)^{\mathcal{A}+p} \models \varphi$.

in other words:

$$
(M, s) \models \dot{\exists} p \varphi \quad \text { iff } \quad(M, s)^{\mathcal{A}+p} \models \varphi
$$

In the last, all implicit knowledge wherein only $p$ was unaware has become explicit.

Variations: forgetting: Continuing on the theme of variations, it seems that bisimulation quantification is essential when becoming awaring but not so when forgetting. Then, the simpler 'reassignment of awareness' version as used just now always suffices. Suppose that forgetting were also a primitive in the language. Then we can define, straightforwardly:

$$
(M, s) \models \exists p \varphi \quad \text { iff } \quad(M, s)^{\mathcal{A}-p} \models \varphi
$$

Variations: speculative knowledge: Finally, consider a version $K_{i}^{A}$ of the knowledge operator defined as follows:

$$
K_{i}^{A} \varphi \quad \text { iff } \quad \forall \operatorname{var}(\varphi) K_{i} \varphi
$$

In other words, $\varphi$ may contain several facts and agents of which the agent is currently unaware, but no matter how she becomes aware of that she will still (explicitly) know that $\varphi$. $(\operatorname{var}(\varphi)$ contains all agent and propositional variables in $\varphi$, also-what we haven't stated but what can be easily proved-successively becoming aware of facts and agents is commutative, therefore $\forall x \forall y$ can be identified with $\forall\{x, y\}$, and so on for any finite subset.)

In the economics literature this - tentatively calledspeculative knowledge $K^{A}$ seems often confused with implicit knowledge. You, agent $i$ who is aware of agent $j$ and fact $p$ but unaware of fact $q$, may speculatively know that: $q \vee \neg q$, or (assuming knowledge) $K_{j} q \rightarrow q$, or $p \wedge q \rightarrow p$. If $q$ were ever to become known, anything involving it derived from validities will become true. On the other hand, $q$ may be implicitly known, but clearly not speculatively.

\section{INDIVIDUAL GLOBAL AWARENESS}

Language: The difference with the language $\mathcal{L}_{0}$ for public global awareness is that the operators $\exists$ and $A$ are now relative to an agent. The language for individual awareness serves both the logic of individual global awareness $L I G A$ and the logic of individual local awareness $L I L A$, to be introduced in the next section.

The language $\mathcal{L}$ of individual awareness is defined as

$$
\varphi:=p|\varphi \wedge \varphi| \neg \varphi\left|K_{i} \varphi\right| \exists_{i} p \varphi\left|\exists_{i} i \varphi\right| A_{i} \varphi
$$

where $p \in P$ and $i \in N$. The abbreviations for explicit knowledge and awareness now are

$$
\begin{aligned}
& \dot{K}_{i} \varphi=A_{i} \varphi \wedge K_{i} \varphi \\
& \dot{\exists}_{i} p \varphi=\neg A_{i} p \wedge \exists_{i} p\left(\varphi \wedge A_{i} \varphi\right) \\
& \dot{\exists}_{i} j \varphi=\neg A_{i} K_{j} \top \wedge \exists_{i} j\left(\varphi \wedge A_{i} K_{j} \top\right) \\
& \dot{\exists}_{i} p \varphi=A_{i} p \wedge \exists_{i} p\left(\varphi \wedge \neg A_{i} p\right) \\
& \dot{\exists}_{i} j \varphi=A_{i} K_{j} \top \wedge \exists_{i} j\left(\varphi \wedge \neg A_{i} K_{j} \top\right)
\end{aligned}
$$

Formula $\dot{\exists}_{i} p \varphi$ stands for 'after (some way in which) agent $i$ becomes aware of atom $p, \varphi$ ', and $A_{i} \varphi$ stands for 'agent $i$ is aware of $\varphi$ ', etc. The free variables of a formula in $\mathcal{L}$ are defined as before with different clauses $\operatorname{var}\left(\exists_{i} p \varphi\right)=$ $\operatorname{var}(\varphi)+i-p, \operatorname{var}\left(\exists_{i} j \varphi\right)=\operatorname{var}(\varphi)+i-j$, and $\operatorname{var}\left(A_{i} \varphi\right)=$ $\operatorname{var}(\varphi)+i$.

Semantics: For each agent, its awareness of other agents and facts is the same throughout a model, and as already introduced we use shorthand $\mathcal{A}_{i}(S)$ for the subset of the relevant facts and visible agents for agent $i$. One could consider further constraints such as self-awareness, 'for all $i \in N, i \in \mathcal{A}_{i}(S)$ ', but we do not require that, as we prefer to keep our approach as general as possible. The crucial clauses in the semantics are as follows (agent forgetting is similar).

$$
\begin{aligned}
& (M, s) \models \exists_{i} p \varphi \quad \text { iff there is a }\left(M^{\prime}, s^{\prime}\right) \text { such that : }
\end{aligned}
$$

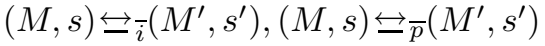

$$
\begin{aligned}
& \text { and }\left(M^{\prime}, s^{\prime}\right) \models \varphi \\
& (M, s) \models \exists_{i} j \varphi \text { iff there is a }\left(M^{\prime}, s^{\prime}\right) \text { such that : }
\end{aligned}
$$

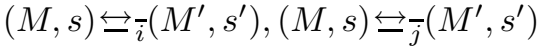

$$
\begin{aligned}
& \text { and }\left(M^{\prime}, s^{\prime}\right) \models \varphi \\
& (M, s) \models A_{i} \varphi \quad \text { iff } \quad \operatorname{var}(\varphi) \subseteq \mathcal{A}_{i}(S)
\end{aligned}
$$

The set of validities (and the logic) is called LIGA (Logic of Individual Global Awareness). The semantics for $\dot{\exists}_{i} p \varphi$ amounts to the requirement that in $(M, s)$ (there is a way such that), after agent $i$ becomes aware of $p, \varphi$ is true, if and only if $\varphi$ remains true in $\left(M^{\prime}, s^{\prime}\right)$ for all agents except $i$ and for all atoms except $p$. Note that this is a

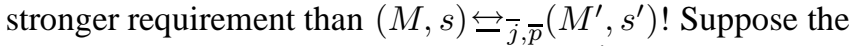
latter were the case, and let $q \neq p$. $M^{\prime}$ may now differ for agent $i$ in the value of $q$-and that would be awkward if $i$ were already aware of $q \ldots$ But if it is required that

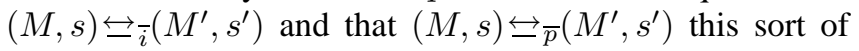
eventuality is ruled out. 
There are open questions on the relation between the logics $L P G A$ and $L I G A$. For example, it is unclear if $A \varphi$ (public awareness!) can be expressed in $\mathcal{L}$, as the obvious infinitary conjunction $\bigwedge_{i \in N} A_{i} \varphi$ is not a formula in the language. As the current agents may always become aware of yet another agent, we cannot restrict the set of all agents to a finite set. We do not know of a logic with our intuitive requirement that the set of agents must be infinite. We have not yet explored the axiomatization of this logic $L I G A$ of individual global awareness-it suffers from the same backdraws for bisimulation quantifiers as $L P G A$. All variations discussed under $L P G A$ apply here too.

\section{INDIVIDUAL LOCAL AWARENESS - AND FURTHER RESEARCH}

We now move to the most complex stage of awareness change. A first observation is that we can keep the same language $\mathcal{L}$ and even the same semantics for the operators as there. The difference is that it applies to a larger class of models, therefore the change of awareness allowed in the bisimulation variation 'except for variable $p$ ' need no longer be global, for all states in the model, but may now be 'almost' local: in the actual state only. Almost: in accordance with the conventions proposed in Section II, 'no uncertain awareness' is an invariant, so, e.g. when the accessibility is an equivalence relation, we only may change the awareness of $p$ in all states of the agent's actual equivalence class (or, therefore, across a union of such classes). So we have a $\operatorname{logic} L I L A$ for individual local awareness change. As also said in Section II, the variation allowed by the part

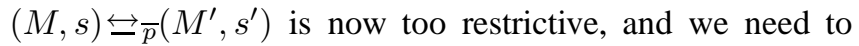
employ more in full the freedom for local variation for other agents. This can be expressed with our alternative notion, of awareness bisimulation. The corresponding basic construct for becoming aware is $\exists_{i}^{A} p \varphi$, with an upper index to distinguish it from the previous $\exists_{i} p \varphi$, where the $A$ expresses that it is interpreted using $\mathfrak{R}^{A}$. Its semantics is:

$$
\begin{aligned}
& (M, s) \models \exists_{i}^{A} p \varphi \text { iff there is a }\left(M^{\prime}, s^{\prime}\right) \text { such that }
\end{aligned}
$$

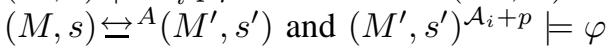

This says that (there is a way in which) the agent $i$ becomes aware of atom $p$ in the current state if there is a model similar to the current one in all its observable aspects except that fact $p$ is added to the awareness set for that agent in all states accessible for that agent from actual states $s^{\prime}$ (in accordance with 'no uncertain awareness'). Given the relation between bisimulation and awareness bisimulation at the end of Section II we think we can easily obtain the following results - but honesty requires us to admit that the submission rush did not give us time to reassuringly prove them (for the second, it is helpful to understand how the combined requirement of $(M, s) \overleftrightarrow{-}\left(M^{\prime}, s^{\prime}\right)$ and

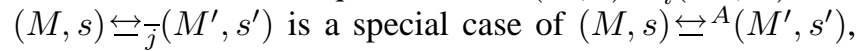
given individual global awareness):
- Awareness bisimilar structures satisfy the same explicit knowledge:

If $(M, s) \overleftrightarrow{\leftrightarrows}^{A}\left(M^{\prime}, s^{\prime}\right)$, then $(M, s) \models \dot{K}_{i} \varphi$ iff

$(M, s) \models \dot{K}_{i} \varphi$ for all $\varphi \in \mathcal{L}$.

- Given individual global awareness, $\exists_{i} p$ and $\exists_{i}^{A} p$ are (explicitly) indistinguishable:

If $(M, s) \models \neg A_{i} p \wedge A_{i} \exists_{i} p \varphi$, then $(M, s) \models \exists_{i} p \varphi$ iff $(M, s) \models \exists_{i}^{A} p \varphi$.

\section{CONCLUSIONS}

The results in this paper are: $(i)$ the logics $L P G A$ and $L I G A$, $(i i)$ a way to model agents becoming aware of other agents, $($ iii $)$ an integrated approach for becoming aware and forgetting, and $(i v)$ the definition of awareness bisimulation: we clearly have not yet explored the use of the latter to the full, but we think this might prove a very useful notion for the community to explore awareness dynamics.

The logics may not be more expressive than epistemic logic. Adding a transitive operator such as common knowledge would greatly increase the expressivity, without losing decidability. The logics seem suited to succinctly express difficult concepts in economics concerning awareness and unawareness. As with anything involving second-order quantification, an axiomatization will remain a challenge but we consider the dynamic semantics of awareness of sufficient, independent, interest, in particular in view of relations with economics. Integration of logics for awareness change with logics for information change is very promising.

\section{ACKNOWLEDGMENT}

This paper explores the approach suggested in Section 6 of [1], entitled 'Becoming aware of factual information'. The LOFT 2008 conference in Amsterdam proved a fertile breeding ground for ideas on awareness and knowledge, and we kindly acknowledge interactions with Oliver Board, Jan Broersen, Brian Hill, Joris Hulstijn, and Giacomo Sillari. In particular we owe Jan Broersen for sharing with us his similar ideas on becoming aware of other agents, and stimulating discussions during the further development of our ideas. The University of Western Australia supported Hans' visit to Tim in December 2008, where the major technical ideas emerged from a four-day non-stop work session.

\section{REFERENCES}

[1] H. van Ditmarsch and T. French, "Simulation and information," in Proceedings of KRAMAS, Sydney, ser. LNAI, J. Broersen and J.-J. Meyer, Eds. Springer, 2009, to appear. Also presented at LOFT 2008, Amsterdam.

[2] R. Fagin and J. Halpern, "Belief, awareness, and limited reasoning," Artificial Intelligence, vol. 34, no. 1, pp. 39-76, 1988.

[3] S. Modica and A. Rustichini, "Unawareness and partitional information structures," Games and Economic Behavior, vol. 27, pp. 265-298, 1999. 
[4] A. Heifetz, M. Meier, and B. Schipper, "Interactive unawareness," Journal of Economic Theory, vol. 130, pp. 78-94, 2006.

[5] T. Agotnes and N. Alechina, "Full and relative awareness: a decidable logic for reasoning about knowledge of unawareness," in Proceedings of TARK XI. New York, NY, USA: ACM, 2007, pp. 6-14.

[6] F. Velazquez-Quesada, "Inference and update," University of Amsterdam, Tech. Rep., 2008, iLLC Research Report PP2008-48.

[7] A. Visser, "Bisimulations, model descriptions and propositional quantifiers," 1996, logic Group Preprint Series 161, Department of Philosophy, Utrecht University.

[8] M. Hollenberg, "Logic and bisimulation," Ph.D. dissertation, University of Utrecht, 1998.

[9] K. Fine, "Propositional quantifiers in modal logic," Theoria, vol. 36(3), pp. 336-346, 1970.

[10] G. Sillari, "Quantified logic of awareness and impossible possible worlds," 2009, to appear in Review of Symbolic Logic.

[11] B. Hill, “Awareness dynamics," 2009, unpublished manuscript.

[12] O. Board and K.-S. Chung, "Object-based unawareness," 2009, unpublished manuscript, prior version presented at LOFT 2006

[13] G. d'Agostino and M. Hollenberg, "Logical questions concerning the mu-calculus: Interpolation, Lyndon and LosTarski," Journal of Symbolic Logic, vol. 65, no. 1, pp. 310332, 2000.

[14] H. van Ditmarsch, W. van der Hoek, and B. Kooi, Dynamic Epistemic Logic, ser. Synthese Library. Springer, 2007, vol. 337.

[15] A. Baltag, L. Moss, and S. Solecki, "The logic of public announcements, common knowledge, and private suspicions," in Proceedings of the 7th Conference on Theoretical Aspects of Rationality and Knowledge (TARK 98), I. Gilboa, Ed., 1998, pp. 43-56. 